\title{
Distributed Optimization For Robot Networks: From Real-time Convex Optimization To Game-Theoretic Self-Organization
}

\author{
Hassan Jaleel and Jeff S. Shamma
}

\begin{abstract}
Recent advances in sensing, communication, and computing technologies have enabled the use of multi-robot systems for practical applications like surveillance, area mapping, and search and rescue. For such systems, a major challenge is to design decision rules that are real-time implementable, require local information only, and guarantee some desired global performance. Distributed optimization provides a framework for designing such local decision making rules for multi-robot systems. In this tutorial, we present a collection of selected results for distributed optimization for robot networks. We will focus on two special classes of problems: (i) real-time path planning for multi-robot systems and (ii) self-organization in multi-robot systems using game-theoretic approaches. For multirobot path planning, we will present some recent approaches that are based on approximately solving distributed optimization problems over continuous and discrete domains of actions. The main idea underlying these approaches is that a variety of path planning problems can be formulated as convex optimization and submodular minimization problems over continuous and discrete action spaces, respectively. To generate local update rules that are efficiently implementable in real time, these approaches rely on approximate solutions to the global problems that can still guarantee some level of desired global performance. For game theoretic self-organization, we will present a sampling of results for area coverage and real-time target assignment. In these results, the problems are formulated as games and online updating rules are designed to enable teams of robots to achieve the collective objective in a distributed manner.
\end{abstract}

\section{INTRODUCTION}

In multi-robot systems, a group of individual robots seeks to achieve a collective objective [1]. Motivating applications include collaborative missions such as exploration [2], area coverage and monitoring [3], [4], task allocation [5], transport [6], and pursuit-evasion [7], [8], as well as self-organization capabilities such as flocking [9], [10], self-assembly [11], [12], [13], and reconfiguration [14], [15].

Beyond enabling tasks that would not be achievable by a single robot, multi-robot architectures have several desirable characteristics. The deployment of multiple robots can execute a mission with more efficiency, e.g., as measured by time, energy, cost, etc. Multi-robot architectures provide resiliency to breakdowns or even adversarial attacks through redundancy

H. Jaleel is with the Intelligent Machines and Sociotechnical Systems (iMaSS) Lab, Department of Electrical Engineering, Syed Babar Ali School of Science \& Engineering at LUMS, Lahore, Pakistan. J.S. Shamma is with the Robotics, Intelligent Systems \& Control (RISC) Lab, Computer, Electrical and Mathematical Sciences and Engineering Division (CEMSE) at King Abdullah University of Science and Technology (KAUST), Thuwal 23955-6900, Saudi Arabia. Emails: hassan.jaleel@lums.edu.pk, jeff.shamma@kaust.edu.sa and the avoidance of a single point of failure. With modular and heterogenous components, multi-robot systems can have broad operational capabilities in different environments. Furthermore, multi-robot architectures can be scalable through the induced emergence of complex behaviors through the programming of simple components.

This paper presents two approaches for multi-robot coordination. The first approach is real-time convex optimization. A variety of problems in multi-robot coordination can be formulated as a convex optimization. We will illustrate how problems such as multi-robot path planning and pursuit-evasion can be approached as a convex optimization problem. Furthermore, we will discuss how these problems can be modified to enable real-time implementation. Whereas distributed approaches typically discuss rates of convergence to find an optimal solution, real-time implementation shifts the discussion from asymptotic optimality to approximate optimality in favor of timely and computational resource constrained implementation.

The second approach is game-theoretic self-organization, where the interactive robots are embedded in a game. The main idea is to incentivize individual robots through a tailored utility function, and then to endow robots with online adaptation rules that allow them to react to the environment and the behaviors of other robots. These results are illustrated on coverage problems and task assignment.

The remainder of this paper is organized as follows. Section II contains a brief presentation of related literature. Section III presents a general framework along with background on convex optimization, both for continuous domain convex functions and discrete domain submodular functions. Section IV shows how various multi-robot path planning problems can be formulated as distributed convex optimization. Section V goes on to discuss how these methods can be modified to enable real-time implementation. Section VI gives an overview of game-theoretic approaches to robot networks. Section VII contains a discussion the relationship of the various approaches in terms of the general framework. Finally, Section VIII contains perspectives and concluding remarks.

\section{RELATED LiterATURE}

The literature on robot networks, or multi-robot systems, is vast. Here we mention selected works specifically related to distributed optimization or game-theoretic methods for robot networks. Reference [16] utilizes distributed optimization for trajectory optimization with pairwise coupling constraints, 
e.g., stemming from formation maintenance or rendezvous. Reference [17] considers convex optimization for motion coordination of large numbers of robots. As will be discussed, coordination problems can be formulated as in the framework of distributed Model Predictive Control (MPC). Reference [18] uses convex optimization in an MPC setting for multi-vehicle coordination.

While these references focus on trajectory optimization, another area that has utilized convex optimization for robot networks is in cooperative localization [19], which is the problem of collectively estimating the positions and orientations of a group of robots [20], [21]. Convex optimization also has been applied to single robot problems such as time-optimal trajectory computation [22] and simultaneous localization and mapping (SLAM) [23].

Another area of interest is in task allocation, in which a collection of robots are assigned to a collection of tasks while optimizing an underlying objective. A particular instance is formation assignment, where a group of robots is to travel to a collection of final destinations. Reference [24] considers such a scenario with objective functions such as total length traveled or total energy consumed. Using properties of the optimal solution, the paper also presents reassignments as the robots execute the initially optimized trajectories. A related problem was considered in [25], but with uncertain travel times. Reference [26] also considers a task assignment algorithm from the perspective of a market-based or auction algorithm. Reference [27] also addresses assignments to locations, but where the robots are heterogenous in that final destinations are valued differently for each robot. Reference [28] considers a variant that includes constraints on the time of arrival. We note that there is a connection between coverage problems (e.g., [3]) and task assignment. Once can consider a geographic location as a task, and there are more tasks than robots. Accordingly, the objective is to maximize the score (e.g., overall coverage) on an assignment while considering costs (e.g., distance traveled).

Also considered herein is multi-robot pursuit-evasion, where a group of robots seeks to intercept one or more evading robots. References [29] and [30] approach the problem (from the perspective of the pursuers) by jointly minimizing the Voronoi region of a single evader. Reference [31] also considers the single evader case, but with uncertainty on the estimated position of the evader. Lastly, reference [32] considers the multiple pursuer and evader case by making simplifying assumptions on the strategies of the pursuers and then mapping the problem into one of dynamic task assignment.

There is relatively less literature on the utilization of game theory for robot networks (although the aforementioned market-based schemes on assignment can be viewed from the perspective of game theory). An early reference connecting game theory as an approach to robot networks is [33]. Reference [34] takes a game-theoretic approach to coverage problems. Reference [35] utilizes cooperative game theory (specifically, the Nash bargaining solution) for consensus seeking. Reference [36] takes a game-theoretic approach to assignment problems, where multiple complementary robots can be assigned to the same task. A general overview of game theory applied to multiagent problems is presented in [37].

\section{DISTRIBUTED OPTIMIZATION}

\section{A. A General Framework}

We start with a general framework for distributed optimization architectures for multi-agent systems. Suppose a team of $n$ agents is assigned a task like monitoring some area of interest. To accomplish the assigned task, we can formulate it as an optimization problem having the following structure:

$$
x^{*}=\arg \min _{x \in \mathcal{X}} \mathcal{G}(x ; \mathcal{E}),
$$

where $x=\left(x_{1}, x_{2}, \ldots, x_{n}\right)$ are the decisions of $n$ agents, $\mathcal{X}$ is the set of allowable decisions, $\mathcal{G}(\cdot ; \mathcal{E})$ is a performance measure that needs to be minimized, and $\mathcal{E}$ is a specification of the associated environment. For example, in a coverage problem, $x_{i}$ represents the location of a mobile sensor in a two or three dimensional region, $\mathcal{E}$ describes the environmental conditions (e.g., the coverage area, navigational obstacles, heat map of coverage events, etc.), and $\mathcal{G}(\cdot ; \mathcal{E})$ captures the effectiveness of a set of sensor locations $x$ under the environmental conditions $\mathcal{E}$. Depending on the formulation, the environment may be broken down into components, e.g., $\mathcal{E}=\mathcal{E}_{1} \cup \mathcal{E}_{2} \cup \ldots \cup \mathcal{E}_{n}$, where $\mathcal{E}_{i}$ represents the initial information of agent $i$ about the overall environment.

In the case of a centralized decision architecture, a single actor has full environmental information, $\mathcal{E}$, and full authority to optimize the decision, $x$, on behalf of all the agents, to ultimately compute (setting aside issues of computational complexity) and implement the optimal decision, $x^{*}$. Likewise, if all agents had access to $\mathcal{E}$, then conceptually, the optimal decision could be computed in parallel among the different agents.

The absence of common shared information of $\mathcal{E}$ and lack of authority over the decisions of other agents motivates the introduction of distributed algorithms. A generic representation is as follows. There is an underlying iterative process that occurs over stages $t=0,1,2, \ldots$ Each agent maintains an evolving local estimate, $\hat{x}^{i}(t)$, of the optimal decision vector, with the intended outcome

$$
\lim _{t \rightarrow \infty} \hat{x}^{i}(t)=x^{*} .
$$

The distributed algorithm specifies how $\hat{x}^{i}(t)$ evolves in response to messages received from other agents and sensory inputs. That is, at each stage, $t$, and for each agent, $i$,

$$
\begin{aligned}
& \mathcal{I}_{i}(t+1)=\mathcal{F}\left(\mathcal{I}_{i}(t), \mathcal{M}_{i}(t), \mathcal{S}_{i}(t)\right) \text { and } \\
& \hat{x}^{i}(t+1)=\mathcal{H}\left(\mathcal{I}_{i}(t), \mathcal{M}_{i}(t), \mathcal{S}_{i}(t)\right),
\end{aligned}
$$

where $\mathcal{I}_{i}(t)$ is an internal state reflecting the $i^{\text {th }}$ agent's processed information, $\mathcal{M}_{i}(t)$ are the messages at stage $t$ received from other agents, and $\mathcal{S}_{i}(t)$ are its sensory inputs at stage $t$. The presumption is that the lack of shared knowledge of $\mathcal{E}$ can be overcome through the combination of online measurements by individual agents and message passing between agents, which is a common feature of distributed optimization algorithms. Unspecified here is an associated graph structure that determines when agents have communication connectivity.

The details of a distributed algorithm are specified by the mappings $\mathcal{F}(\cdot)$ and $\mathcal{H}(\cdot)$. One performance measure is the 
asymptotic optimality of $\hat{x}^{i}(t)$. Other performance measures include rate of convergence, computational burden, communication burden, resilience to failures or adversarial disruptions, and persistence under drifting environment.

The forthcoming Section VII will discuss how the various algorithms presented herein can be viewed in the context of this architecture.

\section{B. Background: Distributed Convex Optimization}

In distributed convex optimization, our starting point is the optimization problem for $n$ agents expressed as

$$
x^{*}=\arg \min _{x \in \mathcal{X}} \mathcal{G}(x ; \mathcal{E})=\sum_{i=1}^{n} g_{i}\left(x ; \mathcal{E}_{i}\right),
$$

where the functions $g_{i}\left(\cdot ; \mathcal{E}_{i}\right)$ are convex for all $i$. Each $g_{i}\left(\cdot ; \mathcal{E}_{i}\right)$ represents a local performance measure of agent $i$, which is not known to any other agent. As previously mentioned, an agent knows its own subset, $\mathcal{E}_{i}$, of the overall environment, $\mathcal{E}=\mathcal{E}_{1} \cup$ $\ldots \cup \mathcal{E}_{n}$. A motivation for distributed optimization is that it may be impractical or undesirable to either disseminate or send to a central processing unit this local information, e.g., because of communication overhead, scalability, or privacy issues. In the standard setup, it is assumed that $g_{i}(\cdot)$ depends on the entire $x$, i.e., the local cost of each agent depends on the decisions of all the agents. A more general setting would be to assume that $g_{i}(\cdot)$ depends on the decisions of a subset of agents.

There are numerous distributed optimization algorithms that can guarantee asymptotic optimality of $\hat{x}_{i}$, i.e., $\lim _{t \rightarrow \infty} \hat{x}^{i}(t)=x^{*}$ (see e.g., [38] and [39] and the references therein for details on distributed optimization algorithm). Two important distributed optimization algorithms in multi-robot literature are dual decomposition based distributed optimization [40] and consensus based distributed optimization [41] and [42]. Both of these algorithms are first order algorithms based on subgradient descent. The advantage of subgradient based methods is that they can be applied to non-smooth convex functions. We omit details herein for space considerations, as distributed convex optimization is discussed extensively in the literature.

\section{Background: Distributed Submodular Minimization}

In some settings, the underlying decision space is discrete, e.g., in diverse application domains like economies of scale, resource allocation, large scale machine learning problems, influence maximization, and utility design for multiagent systems ([43], [44], [45], and [46]). Whereas most distributed optimization literature has addressed convex formulations, it was shown that subgradient based convex optimization algorithms can also be employed for the special case of minimization of submodular functions [47]. Submodular functions are discrete analog of convex functions because their minima can be computed in polynomial time ([48] and [49]). The connection to convex optimization then enables an implementation of distributed algorithms that are derived from distributed convex optimization [50].

In [50], a framework was proposed for the distributed minimization of submodular functions. This framework utilized an equivalent convex reformulation of the submodular minimization problems defined over lattices [47]. In a multiagent setting, there are $n$ agents and each agent is responsible for minimizing its local function $g_{i}(\cdot)$. The optimization problem is

$$
\min _{x \in \mathcal{X}} \sum_{i=1}^{n} g_{i}\left(x ; \mathcal{E}_{i}\right)
$$

where the cost functions $g_{i}\left(\cdot ; \mathcal{E}_{i}\right)$ are submodular and the sets $X_{i}$ are lattices for all $i$. The sum of submodular functions is also submodular [49], which implies that the problem is a submodular minimization problem over lattice product $\mathcal{X}$.

The authors in [47] presented a continuous relaxation for functions defined over lattices that can be computed in polynomial time through a greedy algorithm. This relaxation was based on Lovász extension [49], which was defined for set functions only. The relaxed function was convex if and only if the original function was submodular. Moreover, a minimizer of the submodular function could be computed directly from the minimizer of its convex extension. Thus, an optimal solution to a submodular minimization problem can be computed by solving a convex optimization problem. See [47] and [50] for details.

\section{Multi-Robot PATh Planning}

\section{A. Setup}

In multi-robot path planning, a typical objective is to compute optimal robot trajectories to accomplish some assigned task in a time or energy efficient manner. The assigned task can be for the robots to move in some particular geometric formation, or to monitor an area of interest persistently, or to cover an area for search and rescue mission. In this setup, each robot has a set of feasible control actions $U_{i}$ and the state of a robot evolves according to some dynamical model

$$
x_{i}(k+1)=f_{i}\left(x_{i}(k), u_{i}(k)\right), u_{i}(k) \in U_{i} .
$$

Let $x(k)=\left(x_{1}(k), \ldots, x_{n}(k)\right)$ and $u(k)=$ $\left(u_{1}(k), \ldots, u_{n}(k)\right)$ be the states and inputs at time $k$. Let $x(k)=\left(x_{i}(k), x_{-i}(k)\right)$ and $u(k)=\left(u_{i}(k), u_{-i}(k)\right)$ be agent specific representations of state and control vectors, where $x_{-i}(k)=\left(x_{1}(k), x_{2}(k), \ldots, x_{i-1}(k), x_{i+1}(k), \ldots, x_{n}(k)\right)$. The neighborhood of an agent $i$ is the set of agents whose decisions directly impact $g_{i}(\cdot)$, and is denoted by the set $N_{i}(k)$. Let $x_{N_{i}}(k)=\left(x_{i_{1}}(k), x_{i_{2}}(k), \ldots, x_{i_{\left|N_{i}\right|}}(k)\right)$ be the vector of states of neighbors of $i$ at time $k$. Thus, given a system with initial conditions $x(0)$, a typical problem in multi-robot path planning can be formulated as

$$
\begin{array}{ll} 
& \min _{u(0), u(1), \ldots} \sum_{i=1}^{n}\left[\sum_{k=0}^{\infty} \alpha^{k} g_{i}\left(x_{i}(k), x_{N_{i}}(k), u_{i}(k)\right)\right], \\
\text { s.t. } \quad & x_{i}(k+1)=f_{i}\left(x_{i}(k), u_{i}(k)\right), \text { and } \\
u(k) \in \mathcal{U} \text { for all } k=0,1, \ldots
\end{array}
$$

Here $\mathcal{U}=\prod_{i=1}^{n} U_{i}, \alpha \in(0,1)$ is a discount factor and $g_{i}(\cdot)$ represents the performance measure of robot $i$ at time $k$.

In the problem formulation (6), the robots have decoupled dynamics but coupled stage costs. In particular, the stage cost 
$g_{i}(\cdot)$ of robot $i$ only depends on the states of its neighbors through $x_{N_{i}}(k)$. This condition on stage cost is often enforced to get problem setups that can be efficiently solved in a decentralized manner. We assume that the communication network topology is connected and remains fixed throughout the operation. The restriction of fixed network topology is prevalent in multi-robot applications. To ensure that the condition is not violated, higher level controllers have been proposed to maintain network connectivity while the lower level controllers are designed to achieve a desired task (see e.g. [51], [52], and [53]).

For dynamic programs with input and state constrains, Model Predictive Control (MPC) based solutions for multiagent systems have received significant research attention over the last two decades ([54], [55], [56], [57], and [58]). However, computing a distributed solution to (6) using MPC requires agents to exchange their trajectories with their neighbors a large number of times at each decision time. Therefore, MPC based solutions for multiagent systems have significant communication and computation overhead, which limits the real-time performance of the system. In a system comprising robots like quadcopters, delay in computing a control action can lead to collision among the agents, which will result in system failure.

\section{B. From Multi-Stage to Single-Stage Optimization}

Let $J_{i}\left(x_{i}(0), x_{-i}(0)\right)$ denote the optimal cost in (6) associated with robot $i$, i.e.,

$$
\min _{u_{i}(0), u_{i}(1), \ldots} \sum_{k=0}^{\infty} \alpha^{k} g_{i}\left(x_{i}(k), x_{N_{i}}(k), u_{i}(k)\right) .
$$

Using the principle of optimality, the multi-stage optimization (6) can be written as a single stage optimization

$$
\begin{aligned}
J_{i}\left(x_{i}, x_{-i}\right) & =\min _{u_{i} \in U} g_{i}\left(x_{i}, x_{N_{i}}, u_{i}\right)+\alpha J_{i}\left(x_{i}^{+}, x_{-i}^{+}\right), \\
\text {s.t. } x_{i}^{+} & =f\left(x_{i}, u_{i}\right) .
\end{aligned}
$$

Furthermore, if the performance measure $g_{i}(\cdot)$ is convex and dynamics (5) are linear, then the optimal cost to go $J_{i}(\cdot)$ is also convex [59]. There is a partial decoupling in that the stage cost $g_{i}(\cdot)$ was a function of $x_{i}$ and $x_{N_{i}}$. However, the optimal cost to go $J_{i}(\cdot)$ depends on the entire state vector $\left(x_{i}, x_{-i}\right)$. The reason is that $J_{i}(\cdot)$ represents optimal cost over infinite horizon. Since the network is connected, optimal trajectories over infinite horizon will depend on the actions of all the robots in the system. The resulting problem at the global level is a single-stage convex optimization problem with a partially decomposed cost structure. We say that the resulting problem is single-stage because we are computing the current control $u_{i}$ only instead of an action trajectory over an entire future.

Thus, the original problem is transformed into an equivalent distributed convex optimization problem. This step results in significant reduction in communication overhead since the agents will not have to communicate entire trajectories for computing their actions. Computing optimal cost to go for a dynamic program is often not possible because of the curse of dimensionality. A standard practice at this stage is to use

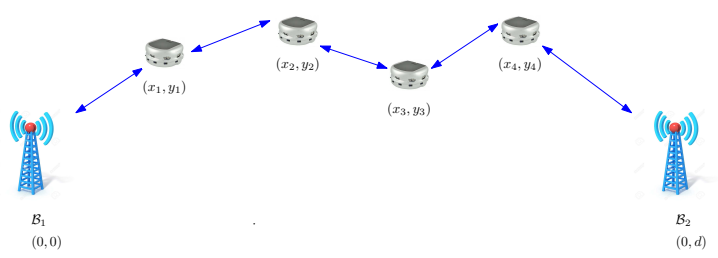

Fig. 1: An illustration of communication network with two base stations and four relay nodes in a two dimensional plane [66].

an approximate cost to go $\tilde{J}$ that can be computed easily and can well approximate the optimal cost to go (cf., approximate dynamic programming [60], [61]).

\section{Real-time Path Planning: Examples}

In this section, we present some examples of multi-robot path planning and the application of the real-time path planning framework presented in the previous section.

\section{A. Co-optimization of Mobility and Communication}

1) Setup: One particular application of multiagent path planning is optimal placement of mobile relay nodes for establishing a communication infrastructure ([62], [63], [64], and [65]). Suppose we have multiple base stations located at different geographic locations, and these base stations need to communicate with each other continuously for an extended period of time $T$. The power required to transmit data over a distance $d$ is proportional to square of the distance.

To reduce the overall energy consumption, one approach is to use a relay network. Suppose a relay network comprising $n_{r}$ mobile relay nodes is deployed in the field. These relay nodes are deployed initially at some locations that are convenient for deployment but are not optimal with respect to energy consumption. At the time of deployment, a network topology is also setup for the overall network that has $n_{b}$ base stations and $n_{r}$ relay nodes, as shown in Fig. 1. This network topology determines which nodes can communicate with each other. Once the relay nodes are deployed, the communication between the base station starts. Thus, the mobile robots have to compute optimal trajectories to optimal locations in a distributed manner while relaying the data between the two base stations. This setup is considered primarily to highlight the challenges associated with real-time implementation of distributed algorithms. The objective is to design a strategy that takes into account the costs of both mobility and communication and can drive the relays to a configuration where the total energy consumption is minimized.

The stage cost for each relay node was a combination of communication cost and mobility cost and had the following structure:

$$
\begin{aligned}
g(x(k), u(k)) & =g_{\mathrm{comm}}(x(k))+g_{\mathrm{mob}}(u(k)), \\
g_{\mathrm{comm}}(x(k)) & =\frac{1}{2} \sum_{i=1}^{n_{r}} \sum_{j \in N_{i}} \kappa_{C}\left\|x_{i}(k)-x_{j}(k)\right\|^{2} \\
g_{\mathrm{mob}}(u(k)) & =\sum_{i=1}^{n_{r}} \kappa_{M}\|u(k)\|^{2} .
\end{aligned}
$$


where $\kappa_{C}$ and $\kappa_{M}$ are constants of proportionality for communication and mobility costs.

With these definitions, the resulting optimization takes the form of a standard linear quadratic regulator ( $\mathrm{LQR}$ ) problem. An LQR problem in discrete time is a dynamic program with quadratic stage cost and linear system dynamics. For multiagent systems, LQR is important because a variety of motion planning problems for multiagent systems like consensus, rendezvous, formation control, optimal placement of mobile relay platforms etc, have can be formulated as LQR problems (see for instance [54], [56], [58], [66], and [67]).

As discussed in Section IV-B, the associated single-stage optimization is

$$
\begin{array}{ll}
\quad \min _{u} x^{\mathrm{T}} Q x+u^{\mathrm{T}} R u+\alpha J\left(\mathrm{x}^{+}\right) \\
\text {s.t. } & x^{+}=A x+B u .
\end{array}
$$

where $J(x)=x^{\mathrm{T}} K x$ and $K$ is the positive definite solution to the Riccati Equation

$$
K=A^{\mathrm{T}}\left(\alpha K-\alpha^{2} K B\left(\alpha B^{\mathrm{T}} K B+R\right)^{-1} B^{\mathrm{T}} K\right) A+Q .
$$

2) Real-time Approximate Implementation: It was shown in [66] for integrator dynamics ( $A=I$ and $B=I$, where $I$ is an identity matrix of appropriate dimension), that all the entries of the matrix $K$ in (10) are non-zero, which implies that the cost of all the agents are coupled. To impose the constraints of communication network topology on the cost structure, we need an approximation of the optimal cost matrix $K$. The approximate matrix $K_{L}$ should satisfy

$$
\left(K_{L}\right)_{i j}= \begin{cases}* & (i, j) \in E \\ 0 & (i, j) \notin E .\end{cases}
$$

Here "*" implies any value that can be non-zero. Computation of approximate cost to go can be formulated as a constrained optimization problem:

$$
\begin{gathered}
K_{L}=\underset{M}{\arg \min }\|K-M\|_{p}, \\
\text { s.t. } M \in \mathcal{K} \text { and } M_{i j}=0 \text { if }(i, j) \notin E .
\end{gathered}
$$

Here $p$ can be any norm depending on the application and $\mathcal{K}$ is the set of all symmetric and positive semidefinite matrices of proper dimension. The approximate one stage optimization problem is

$$
\begin{array}{ll} 
& \min _{u} x^{\mathrm{T}} Q x+u^{\mathrm{T}} R u+\alpha \tilde{J}\left(\mathrm{x}^{+}\right) \\
\text {s.t. } & x^{+}=A x+B u .
\end{array}
$$

where $\tilde{J}(x)=x^{\mathrm{T}} K_{L} x$. The closed loop system resulting from the greedy policy is

$$
\hat{\mu}(x)=-\left[\alpha\left(\alpha B^{\mathrm{T}} K_{L} B+R\right)^{-1} B^{\mathrm{T}} K_{L} A\right] x,
$$

and its stability was proved under certain conditions on system and cost matrices [66]. Moreover, a lower bound for system performance was also computed.

Theorem 5.1: Let $\tilde{J}(x)=x^{T} K_{L} x$ be the approximate cost to go in problem (12) and the resulting greedy policy $\hat{\mu}$ presented in (13) is stable. Let

$$
\epsilon=\left\|\tilde{J}-J^{*}\right\|_{\mathcal{S}^{*}}
$$

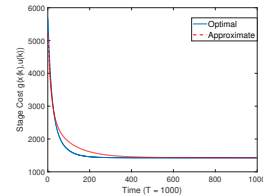

(a)

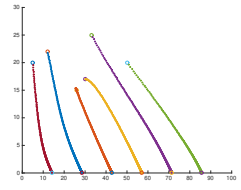

(b)

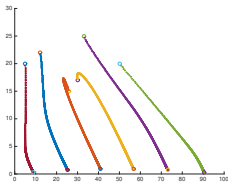

(c)
Fig. 2: Comparisons between exact and approximated implementation for co-optimization of mobility and communication. (a) Evolution of the stage cost. (b) Trajectories under optimal control. (c) Trajectories under sub-optimal control.

and $J^{\hat{\mu}}=\lim _{k \rightarrow \infty} T_{\hat{\mu}}^{k} \tilde{J}$. Then the maximum error between the global optimal solution and the approximate solution is

$$
\left\|J^{\hat{\mu}}-J^{*}\right\|_{\mathcal{S}_{\hat{\mu}}} \leq \frac{2 \alpha \epsilon}{1-\alpha}
$$

In this result, the maximum error between the optimal cost $J^{*}(\cdot)$ and the cost under suboptimal policy $\hat{\mu}$ is expressed in terms of $\epsilon$, which is the error between $J^{*}$ and the approximate cost to go. Better the approximation, lower will be the resulting error. The sets $\mathcal{S}^{*}$ and $\mathcal{S}_{\hat{\mu}}$ are invariant sets under the policies $\mu^{*}$ and $\hat{\mu}$.

To verify the performance of the proposed approximation approach, the mobile relay problem was simulated in MATLAB in which the relay nodes computed their update actions based on the offline-online strategy. The two base stations were located at points $(0,0)$ and $(0,100)$ separated by a distance of 100 units. Six relay nodes per deployed in a two dimensional plane between the two points. The problem in (8) was formulated as an infinite horizon discounted LQR problem with $\alpha=0.99$. The resulting optimal policy from (9) and approximate policy (13) were implemented and the comparison is shown in Fig. 2a. Moreover, the resulting trajectories of the relay nodes under the optimal and approximate policies are presented in Figs. 2b and 2c, respectively. For approximate policy, dual decomposition based distributed optimization algorithm as presented in [40] was implemented with just two iterations of the algorithm for computing an action. The approximate cost matrix had the following structure:

$$
\left(K_{L}\right)_{i j}= \begin{cases}K_{i j} & : i \neq j \text { and }(i, j) \in E \\ K_{i i}-\sum_{p \notin \mathcal{N}_{i}} K_{i p} & : i=j \\ 0 & : \text { otherwise }\end{cases}
$$

where $K_{i j}$ is the $i j$ entry of the optimal cost matrix $K$ defined in (10). It can be seen that for this particular problem, the loss in performance was negligible under approximate policy.

\section{B. Multiagent Pursuit-Evasion Using Linear Programming}

1) Setup: In this section, we present an implementation of a multi-robot system in the setting of a capture the flag game, which is an adversarial game played between two teams: attackers and defenders. The playing arena is a bounded grid in two-dimensional plane. A small region inside the arena is designated as defense zone. The objective of the attackers is to enter the defense zone while avoiding the defenders. The objective of the defenders is to stop the attackers from entering the defense zone. The defenders can either capture the 


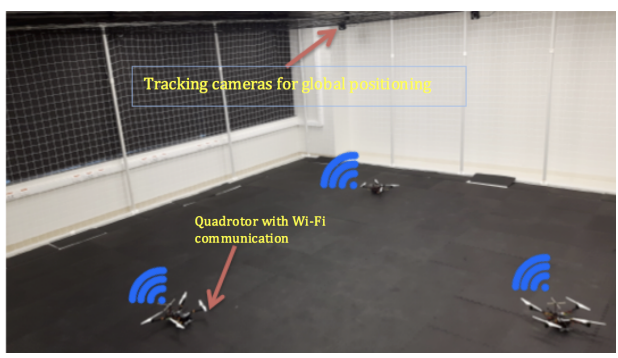

Fig. 3: System configuration for hardware implementation in an indoor environment with customized quadcopters and motion capture system for localization.

attackers or implement a strategy to ensure that no attacker can find a defender free path to the defense zone at any time. An attacker is considered captured if at least one of the defenders is close to its location. There can be obstacles in the arena and the players have to plan there trajectories to avoid collisions with the obstacles as well as collisions with other robots. See Fig. 4 for a configuration of battlefield.

The LP formulation of the capture the flag game as presented in [68] is the following:

$$
\begin{array}{ll} 
& \min _{x_{T_{p}}^{d}}\left[\alpha x_{T_{p}}^{a}+\beta x^{f}\right]^{\mathrm{T}} x_{T_{p}}^{d} \\
\text { s.t. } & x_{T_{p}}^{d}-T_{u} u_{T_{p}}^{d}=T_{x_{0}} x_{i}^{d}(0) \\
& T_{u, c} u_{i, T_{p}}^{d} \leq T_{x_{0}, c} x_{i}^{d}(0)
\end{array}
$$

In this problem setup, $x_{T p}^{d}=\left(x^{d}(1), x^{d}(2), \ldots, x^{d}\left(T_{p}\right)\right)$, and $x_{T p}^{a}=\left(x^{a}(1), x^{a}(2), \ldots, x^{a}\left(T_{p}\right)\right)$, are the trajectories of defenders and attackers respectively over a time horizon $T_{p}$ and $u_{T p}^{d}=\left(u^{d}(0), u^{d}(1), \ldots, u^{d}\left(T_{p}-1\right)\right)$ is the input trajectory for the defenders. In the cost function, the first term corresponds to aggressive behavior of defenders in which they actively pursue the attackers. The second term corresponds to defensive behavior in which they remain close to the defense zone $\left(x^{f}\right)$. The constant $\alpha<0$ and $\beta<0$ are such that $|\alpha|+|\beta|=1$. In the constraints, the first equation correspond to robot dynamics and the inequality correspond to the flow consistency constraints that ensure that the number of defenders leaving a sector cannot be greater than the number of defenders occupying that sector. For collision avoidance, this constraint also ensures that the number of defenders in a sector cannot be greater than one. The matrices $T_{u}, T_{x_{0}}$, and $T_{u_{c}}$ are appropriate matrices defined to represent the constraints over an entire trajectory in terms of the initial conditions only.

For the attacker's trajectory, a diffusive model was assumed in which the attackers were assumed to move towards the defense zone with high probability. The problem was solved in an MPC setting such that at each time $k$ trajectories for the defenders were computed over the interval $\left[k, k+T_{p}-1\right]$ under the assumed model for the attackers. However, only the first instance of the control $u(k)$ was executed to update defenders locations and the problem is resolved at time $k+1$. In the practical implementation, there was a single attacker, which was controlled by a human operator who could see the entire arena. On the other hand, each defender solved the LP, based on its local information, to compute its strategy.

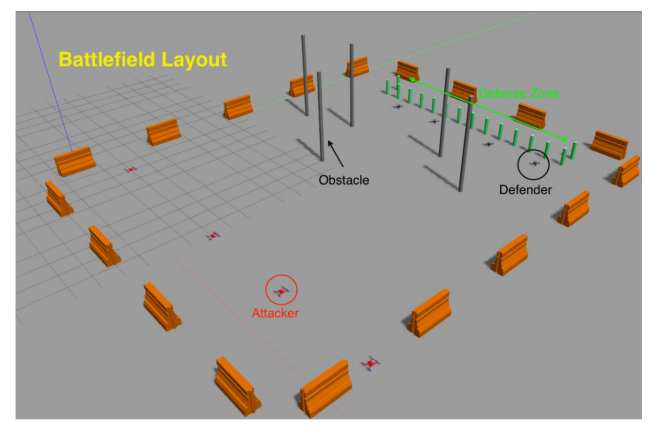

Fig. 4: Representation of a battle field developed in Gazebo. The defense zone is marked by small poles towards the top right. The tall poles represent obstacles in the field. The barriers represent boundary of the arena. The attack and defense team consists of quadcopters such that the defense team is initially deployed in front of the arena and the attackers are deployed on the opposite side [69].

2) Real-time Approximate Implementation: The LP in (15) is a convex optimization, and accordingly can be addressed using methods such as the aforementioned distributed approaches. These approaches require message passing consisting of entire trajectories. Furthermore, these iterations would be done repeatedly in an MPC manner. To alleviate this issue-but at the cost of suboptimality-the problem was modified as follows. The inputs to the LP in (15) are the initial locations of the defending and attacking robots. For practical implementation in [70], we assume that each defending robots internally plans the trajectory for itself and its neighbors, but only executes (the initial portion of) its own trajectory. Accordingly, each defending robot takes the positions of nearby defending robots of its own LP.

This approach was implemented using four custom-built quadcopters. Each quadcopter was equipped with a PX4 autopilot and an Odroid XU4 onboard embedded Linux computer [69]. Moreover, a wireless communication infrastructure was developed for communication among the players and with a base station. The different hardware and software components of the system were managed by Robot Operating System (ROS). The experiment was conducted in a lab environment equipped with OptiTrack motion capture system for localization. The dimensions of the arena were $6 \times 7 \times 3$ meters. Before flight testing, the execution speed of the algorithm was tested on the Odroid XU4 embedded computer for various problem configurations. The results are presented in Table I. These experiments confirmed that the LP based planning algorithm could be implemented efficiently for good real-time performance.

TABLE I: Algorithm execution speed on ODROID XU4

\begin{tabular}{|c|c||c|c|c|}
\hline Setup \# & Sectors & Agents & Prediction steps $\left(T_{p}\right)$ & Freq. $(\mathrm{Hz})$ \\
\hline 1 & $10 \times 10$ & 3 vs. 1 & 3 & 2.3 \\
\hline 2 & $10 \times 10$ & 3 vs. 1 & 2 & 5.5 \\
\hline 3 & $10 \times 10$ & 3 vs. 1 & 1 & 22.7 \\
\hline 4 & $7 \times 7$ & 3 vs. 1 & 3 & 11.0 \\
\hline 5 & $7 \times 7$ & 3 vs. 1 & 2 & 27.8 \\
\hline 6 & $7 \times 7$ & 3 vs. 1 & 1 & 111.0 \\
\hline
\end{tabular}




\section{Multiagent Pursuit-Evasion Using Submodular Minimiza-} tion

Multi-robot path planning over a discrete decision set is a novel application domain of submodular minimization [50]. For multiagent systems, some fundamental behaviors were identified in [71] that can generate complex system behaviors. These simple behaviors included alignment, cohesion, go-togoal, and collision/obstacle avoidance.

Among the fundamental behaviors mentioned above, alignment, cohesion and go-to-goal behaviors can be modeled in the form of attractive potential fields whereas collision and obstacle avoidance require repulsive potential fields. In [50] it was shown that these attractive and repulsive forces can be generated by submodular functions. For instance, given any two robots located at points $x_{i}$ and $x_{j}$ over a two dimensional grid $\mathbf{G}=G \times G$, where $G=\left\{0,1,2, \ldots, n_{G}\right\}$, we define a function

$$
f_{\text {attract }}\left(x_{i}, x_{j}\right)=\left\|x_{i}-x_{j}\right\|_{2}^{2} \text {. }
$$

This function generates an attractive force between the two robots and the function is minimized when $x_{i}=x_{j}$. The function $f_{\text {attract }}(\cdot)$ is submodular with respect to $x_{i}$ and $x_{j}$ over a lattice product [43].

To avoid obstacles in the environment or collision among agents, repulsive potential forces are required. Suppose we have an agent location at $x_{i}=\left(x_{i}^{1}, x_{i}^{2}\right)$ on the grid $\mathbf{G}$. For obstacle and collision avoidance, the following functions were proposed in [50] and verified in real-time simulation environment in [72]:

$$
f_{\mathrm{rep}}^{1}\left(x_{i}, c^{1}\right)=\zeta_{1} e^{-\zeta_{2}\left(x_{i}^{1}-c^{1}\right)^{2}}, f_{\mathrm{rep}}^{2}\left(x_{i}, c^{2}\right)=\zeta_{1} e^{-\zeta_{2}\left(x_{i}^{2}-c^{2}\right)^{2}} .
$$

The function $f_{\text {rep }}^{1}(\cdot)$ generates repulsive forces between an agent located at $x_{i}$ and an entire plane $x_{i}^{1}=c^{1}$. Similarly, $f_{\text {rep }}^{2}(\cdot)$ generates a repulsive force between the agent and an entire plane $x_{i}^{2}=c^{2}$. In these functions $\zeta_{1}$ and $\zeta_{2}$ are positive constants that control the magnitude and rate of decay of repulsive forces. This function, which generates repulsive forces between a point and a plane, was shown to be submodular. Furthermore, it was demonstrated in [72] that these repulsive forces can be effectively combined to ensure collision avoidance and obstacle avoidance. For instance, in Fig. 5a, robots located at $x_{i}$ and $x_{j}$ can avoid collision if they avoid the plane highlighted. Similarly, in Fig. 5b, three robots located at $x_{i}, x_{j}$ and $x_{l}$ can avoid collisions by avoiding the highlighted planes. Although this approach for collision avoidance is conservative but it offers the unifying approach of submodular minimization via subgradient methods for distributed motion planning over a grid structure:

The problem of computing efficient and feasible trajectories for the defense team was formulated as a distributed optimization problem for real-time implementation in [50]. At each time $k$, the online optimization problem had the following structure.

$$
\begin{aligned}
\min _{u^{d} \in \mathcal{U}^{d}} & \sum_{i=1}^{n} g_{i}\left(x^{d}, u^{d},\left(\mathrm{x}^{a}\right)^{+}, x^{f}, x^{\mathrm{obs}}\right) \\
\text { s.t. } & \left(x^{d}\right)^{+}=x^{d}+\mathrm{u}^{d} .
\end{aligned}
$$

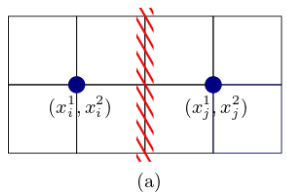

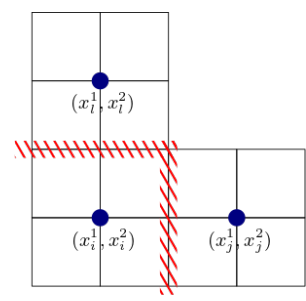

(b)
Fig. 5: Illustration of collision avoidance using submodular repulsive forces.

where $\mathcal{U}^{d}=\prod_{i=0}^{n_{d}-1} U_{i}^{d}$ and $U_{i}^{d}=\{-1,0,1\} \times\{-1,0,1\}$. In this formulation, $g_{i}(\cdot)$ is the local cost of defender $i, x^{d}$ and $x^{a}$ are the state vectors containing the current locations of defenders and attackers respectively and $u^{d}$ is the input vector for the defenders. The vectors $x^{f}$ and $x^{\text {obs }}$ are the locations of the defense zone and the obstacles. The feasible control set of each robot is $\{-1,0,1\} \times\{-1,0,1\}$, i.e., a robot is allowed to move at most one step along horizontal and vertical direction.

In this setup, each defender was preassigned a portion of the defense zone that it had to defend. Therefore, defender $i$ had to remain close to its area of responsibility. However, it also had to pursue any attacker that could potentially attack its area of responsibility. Defenders also had to remain somewhat close to other defenders to act as a team. Finally, each defender had to avoid collisions with obstacles as well as with other defenders. To accomplish all of the above mentioned tasks, the local cost of defender $i$ had the following form:

$$
\begin{aligned}
g_{i}=\alpha_{i}^{f} g_{i}^{f}\left(x_{i}^{d}, u_{i}^{d}, x^{f}\right)+\alpha_{i}^{a} g_{i}^{a}\left(x_{i}^{d}, u_{i}^{d},\left(x^{a}\right)^{+}\right)+ \\
g_{i}^{d}\left(x^{d}, \mathrm{u}^{d}\right)+g_{i}^{\text {avoid }}\left(x^{d}, u_{i}^{d}, x^{\mathrm{obs}}\right),
\end{aligned}
$$

where $\alpha_{i}^{f}>0$ and $\alpha_{i}^{a}>0$ were such that $\alpha_{i}^{a}+\alpha_{i}^{f}=1$. The function $g_{i}^{f}(\cdot)$ generated an attractive force towards a central point of the area of responsibility of $d_{i}$. The defenders knew the exact locations of the defense zone. Similarly, $g_{i}^{a}(\cdot)$ generated an attractive force towards the attacker that was closest to the area of responsibility of $d_{i}$. Since the defender did not know $\left(\mathrm{x}^{a}\right)^{+}$, which is the vector of the locations of attackers at the next time step, it assumed a model for attacker motion to predict its location at the next step. Each defender had a sensing region and it was only be aware of the attackers within its sensing domain.

These attractive and repulsive forces were generated by efficiently utilizing the potential functions defined in (16) and (17). The resulting problem that was solved at each time step was a submodular minimization problem. This decentralized problem was solved in real-time by first formulating the equivalent convex optimization problem and then finding its minimum through consensus based subgradient descent algorithm.

\section{Game-Theoretic Self Organization}

Game theory is the study of interactions between independent and rational decision makers [73]. Over the last decade, there has been a rapid surge in the use of game-theoretic methods for engineering applications like multi-robot path planning, resource allocation, and data 
routing [37]. In this setting, each robot is modeled as a rational decision maker whose objective is to maximize its own utility. However, the utility of an agent depends on the actions of the other agents in the system as well. Therefore, the agents have to negotiate with each other repeatedly to make their decisions. This process of repeated negotiation and decision making is referred to as learning in games ([74], [75], and [76]).

\section{A. Game Setup}

Let $P=\{1,2, \ldots, n\}$ be the set of players, $A_{i}=$ $\left\{a_{1}, a_{2}, \ldots, a_{m_{i}}\right\}$ be the set of actions of player $i$, and $\mathcal{A}=$ $A_{1} \times A_{2} \times \cdots \times A_{n}$. be the set of joint action profiles. Players have preferences over action profiles and these preferences are defined in terms of their utility functions $U_{i}: \mathcal{A} \rightarrow \mathbb{R}$. Player $i$ prefers profile a over $\mathrm{a}^{\prime}$ if $U_{i}(\mathrm{a})>U_{i}\left(\mathrm{a}^{\prime}\right)$. An action profile a is often represented from the perspective of player $i$ as $\mathrm{a}=\left(a_{i}, \mathrm{a}_{-i}\right)$, where $\mathrm{a}_{-i}$ is the actions of all the players in other than $i$ in a. Similarly $\mathcal{A}_{-i}=\prod_{j \neq i} A_{j}$ is the set of joint actions of all the players other than $i$.

Given an action profile $a_{-i}$, the set of best response actions of player $i$ is

$B_{i}\left(\mathrm{a}_{-i}\right)=\left\{a_{i}^{*} \in A_{i} \mid U_{i}\left(\mathrm{a}_{i}^{*}, \mathrm{a}_{-i}\right) \geq U_{i}\left(a_{i}, \mathrm{a}_{-i}\right) \forall a_{i} \in A_{i}\right\}$.

An action profile $a^{*}$ is a Nash Equilibrium (NE), if

$$
U_{i}\left(a_{i}^{*}, \mathrm{a}_{i}^{*}\right) \geq U_{i}\left(\mathrm{a}_{i}, \mathrm{a}_{i}^{*}\right) \text { for all } a_{i} \in A_{i} .
$$

Thus, an action profile is a NE if no player has any incentive to unilaterally change his action. A game can have a unique $\mathrm{NE}$, multiple NE, or no NE at all. A game is a potential game if there exists a function $\phi: \mathcal{A} \rightarrow \mathbb{R}$ such that for every player $i \in P$, for every $\mathrm{a}_{-i} \in \mathcal{A}_{-i}$, and for every $a_{i}^{\prime}$ and $a_{i}^{\prime \prime}$ in $A_{i}$, the following condition is satisfied:

$$
\phi\left(a_{i}^{\prime}, \mathrm{a}_{-i}\right)-\phi\left(a_{i}^{\prime \prime}, \mathrm{a}_{-i}\right)=U_{i}\left(a_{i}^{\prime}, \mathrm{a}_{-i}\right)-U_{i}\left(a_{i}^{\prime \prime}, \mathrm{a}_{-i}\right) .
$$

In the above condition, $\phi$ is a global function. The condition implies that in a potential game, any local improvement in the utility of player $i$ in switching from $a_{i}^{\prime}$ to $a_{i}^{\prime \prime}$ will result in the same improvement in the global potential function [77].

\section{B. Utility Design}

In a multiagent system, the agents are players and the set of possible strategies of a player constitutes its set of actions. Thus, given a global objective for a multiagent system, the first research challenge is to design utility functions for individual players ([78] and [79]). These utility functions should be aligned with the global objective function so that any improvement in the local utility should result in an improvement in the global utility as well. One utility design approach that generates local utility functions that are aligned with global utility is called a Marginal Contribution Utility (MCU) [80].

Let $\mathcal{R}: \mathcal{A} \rightarrow \mathbb{R}$ be the global utility function, which defines the global objective of the system. Then, using MCU, the utility function of player $i$ is defined as follows:

$$
U_{i}\left(a_{i}, \mathrm{a}_{-i}\right)=\mathcal{R}\left(a_{i}, \mathrm{a}_{-i}\right)-\mathcal{R}\left(a_{i}^{0}, \mathrm{a}_{-i}\right) .
$$

where $a_{i}^{0} \in A_{i}$ is called a null action. Thus, the marginal contribution utility of player $i$ is the difference in global utility if player $i$ selects $a_{i}$ versus if player $i$ did not participate $a_{i}^{0}$. If the utility of every player is defined using MCU, then the resulting game is a potential game with potential function $\phi(\mathrm{a})=\mathcal{R}(\mathrm{a})$. In this setup, an action profile that maximizes the potential function is also a global utility maximizer. Thus, for a potential game with marginal contribution utility, global utility maximizer is always a NE.

\section{Learning Mechanisms}

After setting up a game, the next research problem is to design learning mechanisms for individual players that will result in an evolution of action profiles towards some efficient NE. For potential games, a variety of learning mechanisms have been proposed that can guarantee convergence to $\mathrm{NE}$ [74] and [75]. Next, we present two of the learning dynamics that have been used extensively for multi-robot applications.

1) Log-Linear Learning: [81] The following steps are involved in Log-Linear Learning (LLL) at each decision time:

- One player, say player $i$, is selected uniformly at random. All the other players repeat their previous actions, i.e., $\mathrm{a}_{-i}(k)=\mathrm{a}_{-i}(k-1)$

- Player $i$ selects an action $a$ from its action set based on a mixed strategy

$$
a_{i}(k)=a \text { with probability } \frac{e^{U_{i}\left(a, \mathrm{a}_{-i}(k)\right) / T}}{\sum_{a^{\prime} \in A_{i}} e^{U_{i}\left(a^{\prime}, \mathrm{a}_{-i}(k)\right) / T}},
$$

where $T$ is a noise parameter.

When $T$ is infinity, player selects an action with uniform distribution over the action set. When $T$ is zero, player $i$ selects an action from its best response set $B_{i}\left(\mathrm{a}_{-i}\right)$. In general, we are interested in the scenario when $T$ is very small. For small values of $T$, player $i$ selects an action that maximizes its utility with high probability. However, probability of selecting any action other than the best response is also non-zero.

LLL induces a Markov chain over the set of joint action profiles $\mathcal{A}$. This Markov chain is aperiodic and irreducible. For potential games with potential function $\phi$, the unique stationary distribution of this Markov chain is

$$
\pi(\mathrm{a}, T)=\frac{e^{\phi(a) / T}}{\sum_{\mathrm{a}^{\prime} \in \mathcal{A}} \phi\left(\mathrm{a}^{\prime}\right) / T}
$$

The solution concept used to describe the behavior of the system under LLL is stochastic stability. An action profile is stochastically stable if $\pi(\mathrm{a}, T)>0$ as $T \rightarrow 0$. For potential games, only the action profiles that maximize the potential function are stochastically stable. In the marginal contribution utility, global utility function is the potential function. Thus, the only stochastically stable states in the case of potential games with marginal contribution utility are global utility maximizers. 


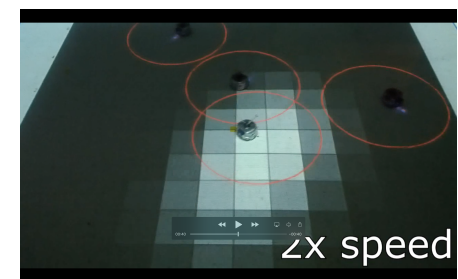

Fig. 6: A screen shot of the multi-robot implementation of coverage setup with BLLL as presented in [83].

2) Regret Matching: [82] This learning dynamics belongs to a class of regret based learning dynamics. At time $k$, the history of the game is

$$
h_{k}=(\mathrm{a}(0), \mathrm{a}(1), \ldots, \mathrm{a}(k-1)),
$$

which is the sequence of all the actions profiles before time $k$. In regret matching, player $i$ selects an action $a_{i}$ based on its retrospective regret for not playing $a_{i}$ at each step in the past. Given a history at time $k$, we define the average regret for not playing $a_{i} \in A_{i}$ as

$$
R_{i}\left(k, a_{i}\right)=\frac{1}{k} \sum_{t=0}^{k-1}\left(U_{i}\left(a_{i}, \mathrm{a}_{-i}(t)\right)-U_{i}(\mathrm{a}(t)) .\right.
$$

Thus, if $R_{i}\left(k, a_{i}\right)$ is positive, player $i$ has a regret of not selecting $a_{i}$ in the past. However, if $R_{i}\left(k, a_{i}\right)$ is negative, the player has no regret for not selecting action $a_{i}$. At each step, player $i$ can compute its average regret recursively as follows:

$R_{i}\left(k+1, a_{i}\right)=\frac{k}{k+1} R_{i}\left(k, a_{i}\right)+\frac{1}{k+1}\left(U_{i}\left(a_{i}, \mathrm{a}_{-i}(k)-U_{i}(\mathrm{a}(k))\right.\right.$.

In regret matching, at each decision time $k$, player $i$ computes its regret vector

$$
R_{i}(k)=\left(R_{i}\left(k, a_{1}\right), R_{i}\left(k, a_{2}\right), \ldots, R_{i}\left(k, a_{m_{i}}\right)\right) .
$$

Then, it selects an action $a_{i}$ with probability $p_{i}\left(k, a_{i}\right)$ where

$$
p_{i}\left(k, a_{i}\right)= \begin{cases}\frac{\left[R_{i}\left(k, a_{i}\right)\right]^{+}}{\mathbb{1}_{m_{i}}^{\mathrm{T}}\left[R_{i}(k)\right]^{+}} & \mathbb{1}_{m_{i}}^{\mathrm{T}}\left[R_{i}(k)\right]^{+}>0, \\ \frac{1}{m_{i}} & \text { otherwise. }\end{cases}
$$

Here $\left[R_{i}\left(k, a_{i}\right)\right]^{+}=\max \left\{R_{i}\left(k, a_{i}\right), 0\right\}, \mathbb{1}_{m_{i}}$ is an $m_{i}$ dimensional vector of all ones, and

$$
\left[R_{i}(k)\right]^{+}=\max \left\{R_{i}(k), \mathbb{O}_{m_{i}}\right\},
$$

where $\mathbb{O}_{m_{i}}$ is an $m_{i}$ dimensional vector of all zero entries. The $l^{\text {th }}$ component of vector $\left[R_{i}(k)\right]^{+}$is equal to the regret for not playing $a_{l}$ if $R_{i}\left(k, a_{l}\right)$ is positive or equal to zero. For potential games under regret matching, the empirical distribution of action profiles converges to a mixed NE. To converge to a pure $\mathrm{NE}$, variations of regret matching were presented in [36].

\section{Application: Distributed Coverage and Task Assignment}

The setup for distributed coverage control presented here is adapted from [84] and its multi-robot implementation was presented in [83]. In this setup, we have a set of locations $L=\left\{l_{1}, l_{2}, \ldots, l_{m}\right\}$, and each location has a weight that signifies the importance of that locations. Let $w_{i}$ be the weight of location $l_{i}$. A set of $n$ mobile sensing agents $(n<m)$ is assigned the task to monitor these locations. These locations are interconnected and the resulting network is represented by a graph $G(V, E)$, where $V$ is the set of locations. The neighborhood of a location $l_{i}$ is the set of all the locations that are directly connected with $l_{i}$, i.e.,

$$
N\left(l_{i}\right)=\left\{l_{j} \in L \mid\left(l_{i}, l_{j}\right) \in E\right\}
$$

The footprint of a sensor positioned at location $l_{i}$ is a set $F\left(l_{i}\right)$, which is the set of all the locations that are covered by the sensor. We assume that $F\left(l_{i}\right)=l_{i} \cup N\left(l_{i}\right)$, which implies that a sensor located at $l_{i}$ covers $l_{i}$ and all the locations in its neighborhood.

To formulate a game, mobile sensors are the players and the set of locations is the set of actions. Let $a_{i}(k-1)$ be the location of player $i$ at time $k-1$. Then, $a_{i}(k) \in F\left(a_{i}(k-1)\right)$, i.e, a mobile node can only move within its footprint at each time step. For a sensor configuration $\mathrm{a}=\left(a_{1}, a_{2}, \ldots, a_{n}\right)$, where $a_{i} \in L$ for each $i \in\{1,2, \ldots, n\}$, the total coverage is

$$
\mathcal{R}(\mathrm{a})=\sum_{s \in F(a)} w_{s}, \text { where } F(\mathrm{a})=\bigcup_{l=1}^{n} F\left(a_{l}\right)
$$

The utility of player $i$ is designed using MCU as defined in (21). For this setup, the null action $a_{i}^{0}$ implies sensing node $i$ does not participate in the sensing operation. Thus,

$$
U_{i}\left(a_{i}, \mathrm{a}_{-i}\right)=\sum_{s \in F\left(a_{i}, \mathrm{a}_{-i}\right)} w_{s}-\sum_{s \in F\left(a_{i}^{0}, \mathrm{a}_{-i}\right)} w_{s},
$$

which is equal to the sum of the weights of the locations that are covered exclusively by player $i$. To compute its utility, player $i$ needs to know the locations in $F\left(a_{i}\right)$ that are covered by other players.

In [84], it was shown that if each player selects its action according to a variant of LLL called Binary LLL (BLLL), then globally optimal configuration of sensing devices will be stochastically stable. The steps involved in BLLL are similar to LLL. At each decision time $k$, one player (say player $i$ ) is randomly selected. All the other players repeat their previous actions. Player $i$ randomly selects an action $a_{i}^{\prime} \neq a_{i}(k-1)$ from $F\left(a_{i}(k-1)\right.$. Then, it switches to $a_{i}^{\prime}$ with probability

$$
p_{i}\left(a_{i}^{\prime}\right)=\frac{e^{U_{i}\left(a_{i}, \mathrm{a}_{-i}(k)\right) / T}}{e^{U_{i}\left(a_{i}, \mathrm{a}_{-i}(k)\right) / T}+e^{U_{i}\left(a_{i}(k-1), \mathrm{a}_{-i}(k)\right) / T}} .
$$

See [84] for numerical comparisons.

In [83], a multi-robot implementation of the coverage problem was presented as shown in Fig. 6. The setup was almost the same as presented here. However, it was assumed that the weights associated with the locations were time varying. It was shown that as long as the weights were slowly varying, global utility maximizing configurations were still stochastically stable.

Similar methods as well as regret matching were applied to task assignment problems, illustrated in Figure 7, [36]. Heterogenous are assigned to different tasks (locations) and there may be benefit in sending complementary packages to the same location. As before, BLLL is guaranteed to spend most of its time at an optimal configuration, whereas regret matching converges to a Nash equilibrium. In the case of 


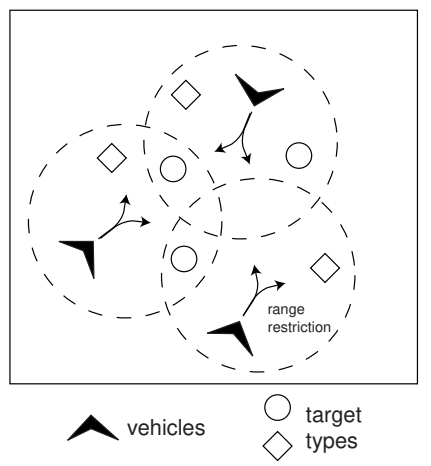

Fig. 7: Setup of vehicle target assignment game from [36]. Each vehicle can service targets within its service area, which is represented by circles.

submodular (here we are talking about maximization versus minimization) scoring functions (i.e., the value of a robot package), then Nash equilibria are a constant factor of $1 / 2$ from the global optimum [85]. See [36] for details and numerical comparisons, which are omitted herein for the sake of brevity.

\section{DISCUSSION}

Section III-A presented a general framework for distributed optimization. Given the diversity of various instantiations, it is impossible to provide a truly general framework without it becoming notationally cumbersome to include multiple special cases. Nonetheless, here is how the various settings presented herein map to this framework. Of particular interest is the impact of real-time implementation considerations in these settings. A key issue in real-time implementation is that the underlying environment (i.e., $\mathcal{E}_{i}$ ), can be measured or discovered gradually in real time and may even be time-varying, in which case the optimization seeks to track a drifting optimal action. Accordingly, it may be necessary to modify standard algorithms to assure real-time convergence or minimize realtime message passing.

In multi-robot path planning Section IV-A, the decision variable of agent $i$ is its trajectory $x_{i}(0), x_{i}(1), x_{i}(2), \ldots$, and the objective is to optimize trajectories with coupled costs in (6). This objective function is rewritten from a dynamic (multistage) form to a static (single stage) form in (7). The decision variable of agent $i$ is now a single control action, $u_{i}$. Given a single stage formulation, various distributed convex optimization algorithms (e.g., Section III-B) can be used. The specific update law of the general framework (2) depends on the underlying algorithm. For example in distributed gradient descent, the evolving algorithm state, $\hat{x}^{i}$, is an estimate of the optimal collective decision, and messages, $\mathcal{M}_{i}(t)$, are shared estimates between agents. Section V-A follows this line of reasoning specialized to an $\mathrm{LQR}$ setting. The environment may be the parameters of the optimization, such as the location of base stations or the communication and mobility cost coefficients. The main concern in Section V-A is how to approximate with guarantees the resulting distributed optimization so that message passing is not required from all agents to all agents.

In the multi-agent pursuit-evasion section of Section V-B, the decision variables are left as trajectories. The environ- ment is the current location of pursuers and evaders. The resulting optimization in (15) is a linear program that can be solved using distributed convex optimization. The realtime consideration in Section V-B is again to introduce an approximate implementation so that the algorithmic message passing is not full trajectories, but rather initial locations. In term of the general framework (2), there is only an initial communication of neighboring locations, but no iterations. Section V-C also deals with multi-agent pursuit-evasion, but in an original discrete form rather than the relaxed convex formulation of Section V-B. Section V-C avoids this relaxation by recognizing an underlying submodular structure to allow the use of distributed convex optimization iterations. Again, the structure of the general framework (2) depends on the specific algorithm being used.

The connection to the general framework is more apparent in the game theoretic formulations. In the case of distributed coverage, the environment is the heat map which is not known a priori. Rather, information about the heat map is obtained online through sensors, or in terms of (2), through sensory information, $\mathcal{S}_{i}(t)$. The messages, $\mathcal{M}_{i}(t)$ are locations of neighboring robots (which alternatively may be sensory input). The evolving decision variable is the position of the mobile robot on grid. Depending on the specific algorithm, there may be auxiliary states as well (e.g., regret vectors). In the setting of task assignments, the environment is the set of tasks available to each agent and the resulting reward associated with collective executions of the task. The decision variable is which task to execute, whose evolution depends on the specific algorithm (e.g., BLLL or regret matching).

We conclude with a discussion on the relationship between the real-time convex optimization and game-theoretic selforganization. Both approaches seek to solve the same problem of distributed optimization in a real-time setting. In distributed convex optimization, there is a natural starting decomposition (which need not be a summation) of the objective function in terms of the summation, $\mathcal{G}(x ; \mathcal{E})=\sum_{i=1}^{n} g_{i}\left(x ; \mathcal{E}_{i}\right)$, and distributed convex optimizations algorithm exploit this structure, along with underlying convexity (or submodularity) properties. This setup can be viewed in the lens of game-theoretic selforganization, where $g_{i}$ is the cost (negative utility) function of agent $i$. In this case, the global optimum is a Nash equilibrium, but there may be Nash equilibria that are not optimal (cf., notions such as price of anarch and price of stability [86], which analyze the overall performance at Nash equilibria). With this association, one can then use gametheoretic algorithms (or their continuous counterparts), but these algorithms do not exploit underlying convexity.

Another distinction in game-theoretic algorithms is the notion of utility design (cf., Section VI-B). In terms of the general framework, there is no decomposition of $\mathcal{G}(x ; \mathcal{E})$. Rather, an important question is how to decompose $\mathcal{G}(x ; \mathcal{E})$ into separate utility functions (or agent incentives) so that the resulting game has a favorable structure in terms in outcome of a learning mechanism [87]. If the resulting utility functions have a convex structure, then one can utilize real-time convex optimization as an alternative to learning mechanisms. Finally, we mention that both settings involve message pass- 
ing/communications, and the communications burden depends on the specific algorithmic instantiation. There is relevant prior work on communications complexity, that provides algorithm independent bounds on messaging requirements for both distributed optimization [88] and game-theoretic learning [89].

\section{CONCLUding Remarks}

We have presented various methodologies for optimization based coordination of robot networks with two complementary approaches. The first is based on distributed optimization, where robots execute a prescribed algorithm of decision making and message passing to assure that the collective decision is one that would have been realized by a centralized planner. The second approach is based on game-theoretic self-organization. Here, a planner prescribes incentives and adaptation rules so that robots self configure into desirable collective configurations. Both approaches only consider a single objective function. However, there are many factors, and ultimately various tradeoffs, that should be considered. These issues also serve as potential future directions for research in this area.

The first is the matter of asymptotic optimality. Both approaches provide guarantees on the eventual outcome. However for applications where time is of the essence, one must trade off optimality with timeliness (and hence suboptimality). We presented different approaches that attempt to address this tradeoff. The main idea was to use distributed optimization as an initial algorithm which is then modified for the sake of real-time implementability but at the cost of asymptotic optimality. In the ideal case, as in Section V-A, it is possible to quantify the loss in performance. A topic for future research is to optimize the level of performance that can be achieved given a fixed time of execution, where the best approach need not be a truncation of an asymptotically optimal iteration. One relevant approach that continues to be explored is the concept of cloud robotics, where computations are split between onboard and off-board platforms (e.g., [90]).

Another consideration is the communication burden of the various algorithms. The presented algorithms for distributed optimization require multiple exchanges of candidate decision vectors. Similar considerations hold in the case of cloud implementations. There has been prior work on the effects of message dropouts or latent information in such algorithms (e.g., an early discussion is in [91]), but these guarantees are again asymptotic and impact the timeliness of execution. Another issue is operating in communication constrained environments, e.g., underwater or underground environments. A future research question is the design of algorithms that admit infrequent or consistently low bandwidth communications.

Yet another consideration is resilience. Two sources of disruptions are individual robot failures and adversarial behaviors. Important research questions are to understand to what degree algorithms are susceptible to either source and how to modify algorithms accordingly to mitigate their impact (e.g., [92]).

There are also non-algorithmic, or physical, considerations in the implementation of these algorithms. One issue is energy consumption. Energy consumption is impacted by computation, communication, and mobility. Typically, mobility would be the largest factor. However computation and communication can become significant with a miniaturization of robot networks, as in swarm robotics (e.g., [93]). Another issue is physical, as opposed to algorithmic, coupling, where robots have physical interactions that impact each other's dynamics. Examples include collaborative transport [6], [94] and selfassembly [14], [15]. Also related to physical implementation is the abstraction that often comes with associated with these algorithms. In order to bring computational tractability, issues such as low level trajectory generation, obstacle avoidance, and collision avoidance may be neglected, which may adversely impact the performance suggested in an abstract representation. Along these lines, there is still relatively little literature that demonstrates these algorithms with fully onboard sensing, computation, communication, and localization.

Finally, we conclude with some comments comparing distributed optimization and game-theoretic approaches. Distributed optimization methods tend to place stronger algorithmic requirements on both individual robots and their coordinated message passing, as compared to the more simplistic hill climbing behaviors of game-theoretic approaches. Such a distinction does not reflect a deficiency of either approach. Rather, it suggests that there should be different expectations in realized performance. Anecdotally, one would expect superior performance from distributed optimization in measures such as collective performance for smaller, perhaps heterogeneous, teams, whereas game-theoretic approaches may excel in metrics such as resilience or swarm (many low cost components) implementation. A future research direction topic is to consider a hybrid approach that may adapt to online conditions. Indeed, the distinction between the two is blurry, and it is sometimes possible to cast one approach in the language of the other (e.g., [95]).

\section{REFERENCES}

[1] T. Arai, E. Pagello, and L. E. Parker, "Guest editorial: Advances in multirobot systems," IEEE Transactions on Robotics and Automation, vol. 18 , no. 5, pp. 655-661, Oct 2002.

[2] W. Burgard, M. Moors, C. Stachniss, and F. E. Schneider, "Coordinated multi-robot exploration," IEEE Transactions on Robotics, vol. 21, no. 3, pp. 376-386, June 2005.

[3] J. Cortes, S. Martinez, T. Karatas, and F. Bullo, "Coverage control for mobile sensing networks," IEEE Transactions on Robotics and Automation, vol. 20, no. 2, pp. 243-255, 2004.

[4] S. L. Smith, M. Schwager, and D. Rus, "Persistent robotic tasks: Monitoring and sweeping in changing environments," IEEE Transactions on Robotics, vol. 28, no. 2, pp. 410-426, April 2012.

[5] G. A. Korsah, A. Stentz, and M. B. Dias, "A comprehensive taxonomy for multi-robot task allocation," The International Journal of Robotics Research, vol. 32, no. 12, pp. 1495-1512, 2013.

[6] N. Michael, J. Fink, and V. Kumar, "Cooperative manipulation and transportation with aerial robots," Autonomous Robots, vol. 30, no. 1, pp. 73-86, Jan 2011.

[7] G. C. Chasparis and J. S. Shamma, "Linear-programming-based multivehicle path planning with adversaries," in Proceedings of the 2005 American Control Conference, June 2005, pp. 1072-1077.

[8] M. Chen, Z. Zhou, and C. J. Tomlin, "Multiplayer reach-avoid games via pairwise outcomes," IEEE Transactions on Automatic Control, vol. 62 , no. 3, pp. 1451-1457, March 2017.

[9] R. Olfati-Saber, "Flocking for multi-agent dynamic systems: algorithms and theory," IEEE Transactions on Automatic Control, vol. 51, no. 3, pp. 401-420, March 2006.

[10] H. G. Tanner, A. Jadbabaie, and G. J. Pappas, "Flocking in fixed and switching networks," IEEE Transactions on Automatic Control, vol. 52, no. 5, pp. 863-868, May 2007. 
[11] E. Klavins, "Programmable self-assembly," IEEE Control Systems Magazine, vol. 27, no. 4, pp. 43-56, August 2007.

[12] M. J. Fox and J. S. Shamma, "Probabilistic performance guarantees for distributed self-assembly," IEEE Transactions on Automatic Control, vol. 60, no. 12, pp. 3180-3194, Dec 2015.

[13] B. Haghighat, E. Droz, and A. Martinoli, "Lily: A miniature floating robotic platform for programmable stochastic self-assembly," in 2015 IEEE International Conference on Robotics and Automation (ICRA), May 2015, pp. 1941-1948.

[14] M. Yim, W. Shen, B. Salemi, D. Rus, M. Moll, H. Lipson, E. Klavins, and G. S. Chirikjian, "Modular self-reconfigurable robot systems [grand challenges of robotics]," IEEE Robotics Automation Magazine, vol. 14, no. 1, pp. 43-52, March 2007.

[15] M. Rubenstein, C. Ahler, N. Hoff, A. Cabrera, and R. Nagpal, "Kilobot: A low cost robot with scalable operations designed for collective behaviors," Robotics and Autonomous Systems, vol. 62, no. 7, pp. 966975, 2014.

[16] S. Bhattacharya, V. Kumar, and M. Likhachev, "Distributed optimization with pairwise constraints and its application to multi-robot path planning," in Proceedings of the Robotics: Science and Systems Conference (RSS 2010), June 2010.

[17] J. C. Derenick and J. R. Spletzer, "Convex optimization strategies for coordinating large-scale robot formations," IEEE Transactions on Robotics, vol. 23, no. 6, pp. 1252-1259, Dec 2007.

[18] F. Farokhi, I. Shames, and K. H. Johansson, Distributed MPC Via Dual Decomposition and Alternative Direction Method of Multipliers. Dordrecht: Springer Netherlands, 2014, pp. 115-131.

[19] E. D. Nerurkar, S. I. Roumeliotis, and A. Martinelli, "Distributed maximum a posteriori estimation for multi-robot cooperative localization," in 2009 IEEE International Conference on Robotics and Automation, May 2009, pp. 1402-1409.

[20] U. A. Khan, S. Kar, and J. M. F. Moura, "Distributed sensor localization in random environments using minimal number of anchor nodes," IEEE Transactions on Signal Processing, vol. 57, no. 5, pp. 2000-2016, May 2009.

[21] S. S. Kia, S. Rounds, and S. Martinez, "Cooperative localization for mobile agents: A recursive decentralized algorithm based on kalmanfilter decoupling," IEEE Control Systems Magazine, vol. 36, no. 2, pp. 86-101, April 2016.

[22] D. Verscheure, B. Demeulenaere, J. Swevers, J. De Schutter, and M. Diehl, "Time-optimal path tracking for robots: A convex optimization approach," IEEE Transactions on Automatic Control, vol. 54, no. 10, pp. 2318-2327, Oct 2009.

[23] M. Liu, S. Huang, G. Dissanayake, and H. Wang, "A convex optimization based approach for pose slam problems," in 2012 IEEE/RSJ International Conference on Intelligent Robots and Systems, Oct 2012, pp. 1898-1903.

[24] M. Turpin, N. Michael, and V. Kumar, "Trajectory planning and assignment in multirobot systems," in Algorithmic Foundations of Robotics $X$, E. Frazzoli, T. Lozano-Perez, N. Roy, and D. Rus, Eds. Berlin, Heidelberg: Springer Berlin Heidelberg, 2013, pp. 175-190.

[25] A. Prorok, "Redundant robot assignment on graphs with uncertain edge costs," in Distributed Autonomous Robotic Systems, N. Correll, M. Schwager, and M. Otte, Eds. Cham: Springer International Publishing, 2019, pp. 313-327.

[26] N. Michael, M. M. Zavlanos, V. Kumar, and G. J. Pappas, "Distributed multi-robot task assignment and formation control," in 2008 IEEE International Conference on Robotics and Automation, May 2008, pp. $128-133$.

[27] E. Montijano and A. R. Mosteo, "Efficient multi-robot formations using distributed optimization," in 53rd IEEE Conference on Decision and Control, Dec 2014, pp. 6167-6172.

[28] S. Chopra, G. Notarstefano, M. Rice, and M. Egerstedt, "A distributed version of the hungarian method for multirobot assignment," IEEE Transactions on Robotics, vol. 33, no. 4, pp. 932-947, Aug 2017.

[29] H. Huang, W. Zhang, J. Ding, D. M. Stipanović, and C. J. Tomlin, "Guaranteed decentralized pursuit-evasion in the plane with multiple pursuers," in 2011 50th IEEE Conference on Decision and Control and European Control Conference, Dec 2011, pp. 4835-4840.

[30] Z. Zhou, W. Zhang, J. Ding, H. Huang, D. M. Stipanović, and C. J. Tomlin, "Cooperative pursuit with voronoi partitions," Automatica, vol. 72, pp. 64-72, 2016.

[31] K. Shah and M. Schwager, "Multi-agent cooperative pursuit-evasion strategies under uncertainty," in Distributed Autonomous Robotic Systems, N. Correll, M. Schwager, and M. Otte, Eds. Cham: Springer International Publishing, 2019, pp. 451-468.
[32] V. R. Makkapati and P. Tsiotras, "Optimal evading strategies and task allocation in multi-player pursuit-evasion problems," Dynamic Games and Applications, vol. 9, no. 4, pp. 1168-1187, 2019.

[33] S. M. LaValle and S. Hutchinson, "Game theory as a unifying structure for a variety of robot tasks," in Proceedings of 8th IEEE International Symposium on Intelligent Control, Aug 1993, pp. 429-434.

[34] M. Zhu and S. Martinez, "Distributed coverage games for energy-aware mobile sensor networks," SIAM Journal on Control and Optimization, vol. 51, no. 1, pp. 1-27, 2013.

[35] E. Semsar-Kazerooni and K. Khorasani, "Multi-agent team cooperation: A game theory approach," Automatica, vol. 45, no. 10, pp. 2205 - 2213, 2009.

[36] G. Arslan, J. R. Marden, and J. S. Shamma, "Autonomous vehicletarget assignment: A game-theoretical formulation," Journal of Dynamic Systems, Measurement, and Control, vol. 129, no. 5, pp. 584-596, 2007.

[37] J. R. Marden and J. S. Shamma, "Game theory and distributed control," in Handbook of Game Theory with Economic Applications. Elsevier, 2015, vol. 4, pp. 861-899.

[38] A. Nedić, A. Olshevsky, and M. G. Rabbat, "Network topology and communication-computation tradeoffs in decentralized optimization," Proceedings of the IEEE, vol. 106, no. 5, pp. 953-976, 2018.

[39] A. Nedić and J. Liu, "Distributed optimization for control," Annual Review of Control, Robotics, and Autonomous Systems, vol. 1, pp. 77103, 2018.

[40] H. Terelius, U. Topcu, and R. M. Murray, "Decentralized multi-agent optimization via dual decomposition," IFAC Proceedings Volumes, vol. 44, no. 1, pp. $11245-11251,2011$.

[41] A. Nedic and A. Ozdaglar, "Distributed subgradient methods for multiagent optimization," IEEE Transactions on Automatic Control, vol. 54, no. 1, pp. 48-61, 2009.

[42] A. Nedic, A. Ozdaglar, and P. A. Parrilo, "Constrained consensus and optimization in multi-agent networks," IEEE Transactions on Automatic Control, vol. 55, no. 4, pp. 922-938, 2010.

[43] D. M. Topkis, "Equilibrium points in nonzero-sum n-person submodular games," Siam Journal on control and optimization, vol. 17, no. 6, pp. 773-787, 1979.

[44] — Supermodularity and Complementarity. Princeton university press, 2011

[45] J. Vondrák, "Optimal approximation for the submodular welfare problem in the value oracle model," in Proceedings of the fortieth annual ACM symposium on Theory of computing. ACM, 2008, pp. 67-74.

[46] J. R. Marden and A. Wierman, "Distributed welfare games," Operations Research, vol. 61, no. 1, pp. 155-168, 2013.

[47] F. Bach, "Submodular functions: From discrete to continuous domains," Mathematical Programming, pp. 1-41, 2018.

[48] J. Edmonds, "Submodular functions, matroids, and certain polyhedra," Edited by G. Goos, J. Hartmanis, and J. van Leeuwen, vol. 11, 1970.

[49] L. Lovász, "Submodular functions and convexity," in Mathematical Programming The State of the Art. Springer, 1983, pp. 235-257.

[50] H. Jaleel and J. S. Shamma, "Distributed submodular minimization and motion planning over discrete state space," IEEE Transactions on Control of Network Systems, 2019.

[51] M. M. Zavlanos, M. B. Egerstedt, and G. J. Pappas, "Graph-theoretic connectivity control of mobile robot networks," Proceedings of the IEEE, vol. 99, no. 9, pp. 1525-1540, 2011.

[52] M. Ji and M. Egerstedt, "Distributed coordination control of multiagent systems while preserving connectedness," IEEE Transactions on Robotics, vol. 23, no. 4, pp. 693-703, 2007.

[53] H. Jaleel and W. Abbas, "A connectivity preserving framework for distributed motion coordination in proximity networks," in 2018 Annual American Control Conference (ACC). IEEE, 2018, pp. 6640-6645.

[54] G. Ferrari-Trecate, L. Galbusera, M. P. E. Marciandi, and R. Scattolini, "Model predictive control schemes for consensus in multi-agent systems with single-and double-integrator dynamics," IEEE Transactions on Automatic Control, vol. 54, no. 11, pp. 2560-2572, 2009.

[55] M. A. Müller, M. Reble, and F. Allgöwer, "Cooperative control of dynamically decoupled systems via distributed model predictive control," International Journal of Robust and Nonlinear Control, vol. 22, no. 12, pp. 1376-1397, 2012.

[56] H. Li and W. Yan, "Receding horizon control based consensus scheme in general linear multi-agent systems," Automatica, vol. 56, pp. 12-18, 2015.

[57] W. B. Dunbar and R. M. Murray, "Distributed receding horizon control for multi-vehicle formation stabilization," Automatica, vol. 42, no. 4, pp. 549-558, 2006. 
[58] T. Keviczky, F. Borrelli, and G. J. Balas, "Decentralized receding horizon control for large scale dynamically decoupled systems," Automatica, vol. 42, no. 12, pp. 2105-2115, 2006.

[59] D. P. Bertsekas, Dynamic Programming and Optimal Control. Athena Scientific Belmont, MA, 1995, vol. 1, no. 2.

[60] Y. Wang, B. O'Donoghue, and S. Boyd, "Approximate dynamic programming via iterated bellman inequalities," International Journal of Robust and Nonlinear Control, vol. 25, no. 10, pp. 1472-1496, 2015.

[61] D. P. Bertsekas, Dynamic programming and optimal control 3rd edition, volume II. Citeseer, 2011.

[62] D. K. Goldenberg, J. Lin, A. S. Morse, B. E. Rosen, and Y. R. Yang, "Towards mobility as a network control primitive," in Proceedings of the 5th ACM international symposium on Mobile ad hoc networking and computing. ACM, 2004, pp. 163-174.

[63] F. El-Moukaddem, E. Torng, and G. Xing, "Mobile relay configuration in data-intensive wireless sensor networks," IEEE Transactions on Mobile computing, vol. 12, no. 2, pp. 261-273, 2011.

[64] H. Jaleel, Y. Wardi, and M. Egerstedt, "Minimizing mobility and communication energy in robotic networks: An optimal control approach," in 2014 American Control Conference. IEEE, 2014, pp. 2662-2667.

[65] Y. Yan and Y. Mostofi, "To go or not to go: On energy-aware and communication-aware robotic operation," IEEE Transactions on Control of Network Systems, vol. 1, no. 3, pp. 218-231, 2014.

[66] H. Jaleel and J. S. Shamma, "Design of real-time implementable distributed suboptimal control: An LQR perspective," IEEE Transactions on Control of Network Systems, vol. 5, no. 4, pp. 1717-1728, 2017.

[67] R. M. Murray, "Recent research in cooperative control of multivehicle systems," Journal of Dynamic Systems, Measurement, and Control, vol. 129 , no. 5, pp. 571-583, 2007.

[68] G. C. Chasparis and J. S. Shamma, "LP-based multi-vehicle path planning with adversaries," Cooperative Control of Distributed MultiAgent Systems, pp. 261-279, 2008.

[69] M. Abdelkader, Y. Lu, H. Jaleel, and J. S. Shamma, "Distributed real time control of multiple uavs in adversarial environment: Algorithm and flight testing results," in 2018 IEEE International Conference on Robotics and Automation (ICRA). IEEE, 2018, pp. 6659-6664.

[70] M. Abdelkader, H. Jaleel, and J. S. Shamma, "A distributed framework for real time path planning in practical multi-agent systems," IFACPapersOnLine, vol. 50, no. 1, pp. 10626-10631, 2017.

[71] C. W. Reynolds, "Flocks, herds and schools: A distributed behavioral model," ACM SIGGRAPH computer graphics, vol. 21, no. 4, pp. 25-34, 1987.

[72] H. Jaleel, M. Abdelkader, and J. S. Shamma, "Real-time distributed motion planning with submodular minimization," in 2018 IEEE Conference on Control Technology and Applications (CCTA). IEEE, 2018, pp. 885-890.

[73] M. J. Osborne and A. Rubinstein, A course in game theory. MIT press, 1994.

[74] D. Fudenberg, F. Drew, D. K. Levine, and D. K. Levine, The Theory of Learning in Games. MIT press, 1998, vol. 2.

[75] H. P. Young, Strategic Learning and its Limits. OUP Oxford, 2004.

[76] J. R. Marden and J. S. Shamma, "Game-theoretic learning in distributed control," Handbook of Dynamic Game Theory, pp. 511-546, 2018.

[77] D. Monderer and L. S. Shapley, "Potential games," Games and Economic Behavior, vol. 14, no. 1, pp. 124-143, 1996.

[78] J. R. Marden and A. Wierman, "Distributed welfare games," Operations Research, vol. 61, no. 1, pp. 155-168, 2013

[79] — - "Overcoming the limitations of utility design for multiagent systems," IEEE Transactions on Automatic Control, vol. 58, no. 6, pp. 1402-1415, 2013.

[80] K. Tumer and D. Wolpert, "A survey of collectives," in Collectives and the Design of Complex Systems. Springer, 2004, pp. 1-42.

[81] L. E. Blume, "The statistical mechanics of strategic interaction," Games and Economic Behavior, vol. 5, no. 3, pp. 387-424, 1993.

[82] S. Hart and A. Mas-Colell, "A simple adaptive procedure leading to correlated equilibrium," Econometrica, vol. 68, no. 5, pp. 1127-1150, 2000.

[83] Y. Lim and J. S. Shamma, "Robustness of stochastic stability in game theoretic learning," in 2013 American Control Conference. IEEE, 2013, pp. 6145-6150.

[84] A. Y. Yazıcıoğlu, M. Egerstedt, and J. S. Shamma, "Communicationfree distributed coverage for networked systems," IEEE Transactions on Control of Network Systems, vol. 4, no. 3, pp. 499-510, 2016.

[85] D. Grimsman, M. S. Ali, J. P. Hespanha, and J. R. Marden, "The impact of information in distributed submodular maximization," IEEE Transactions on Control of Network Systems, vol. 6, no. 4, pp. 13341343, Dec 2019.
[86] G. Christodoulou and E. Koutsoupias, "The price of anarchy of finite congestion games," in Proceedings of the Thirty-Seventh Annual ACM Symposium on Theory of Computing, ser. STOC '05. New York, NY, USA: Association for Computing Machinery, 2005, pp. 67-73.

[87] J. R. Marden and A. Wierman, "Overcoming the limitations of utility design for multiagent systems," IEEE Transactions on Automatic Control, vol. 58, no. 6, pp. 1402-1415, 2013.

[88] A. Xu and M. Raginsky, "A new information-theoretic lower bound for distributed function computation," in 2014 IEEE International Symposium on Information Theory, June 2014, pp. 2227-2231.

[89] Y. Babichenko and A. Rubinstein, "Communication complexity of approximate nash equilibria," in Proceedings of the 49th Annual ACM SIGACT Symposium on Theory of Computing, ser. STOC 2017. New York, NY, USA: Association for Computing Machinery, 2017, pp. 878889.

[90] R. Arumugam, V. R. Enti, L. Bingbing, W. Xiaojun, K. Baskaran, F. F. Kong, A. S. Kumar, K. D. Meng, and G. W. Kit, "Davinci: A cloud computing framework for service robots," in 2010 IEEE International Conference on Robotics and Automation, May 2010, pp. 3084-3089.

[91] D. P. Bertsekas and J. N. Tsitsiklis, Parallel and distributed computation: numerical methods. Prentice hall Englewood Cliffs, NJ, 1989, vol. 23.

[92] H. J. LeBlanc, H. Zhang, X. Koutsoukos, and S. Sundaram, "Resilient asymptotic consensus in robust networks," IEEE Journal on Selected Areas in Communications, vol. 31, no. 4, pp. 766-781, April 2013.

[93] H. Hamann, Swarm Robotics: A Formal Approach. Springer International Publishing, 2018.

[94] C. Gabellieri, M. Tognon, L. Pallottino, and A. Franchi, "A study on force-based collaboration in flying swarms," in Swarm Intelligence, M. Dorigo, M. Birattari, C. Blum, A. L. Christensen, A. Reina, and V. Trianni, Eds. Cham: Springer International Publishing, 2018, pp. $3-15$.

[95] N. Li and J. R. Marden, "Decoupling coupled constraints through utility design," IEEE Transactions on Automatic Control, vol. 59, no. 8, pp. 2289-2294, Aug 2014.

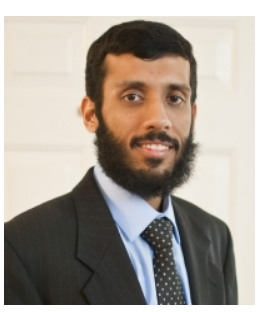

Hassan Jaleel Hassan Jaleel is an Assistant Professor of the Department of Electrical Engineering at the Lahore University of Management Sciences (LUMS) in Lahore, Pakistan. He received his M.S. and Ph.D. degrees in Electrical and Computer Engineering (ECE) with specialization in Systems and Control from the Georgia Institute of Technology, Atlanta, GA, USA. Prior to joining LUMS, Jaleel was a Postdoctoral Research Fellow at the King Abdullah University of Science and Technology (KAUST). His research interests are in the areas of complex networks, game theory, stochastic geometry, and online distributed optimization. Typical application domains of his research are sensor networks, swarm robotics, and irrigation networks.

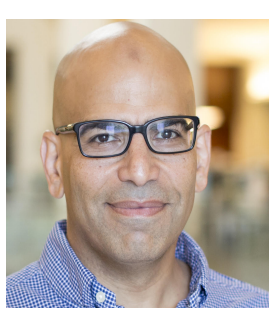

Jeff Shamma Jeff S. Shamma is a Professor of Electrical Engineering at the King Abdullah University of Science and Technology (KAUST). He received a Ph.D. in systems science and engineering from MIT in 1988. Shamma is the former Julian T. Hightower Chair in Systems \& Control in the School of Electrical and Computer Engineering at Georgia Tech, and he has held faculty positions at the University of Minnesota, The University of Texas at Austin, and the University of California, Los Angeles. He is the recipient of an NSF Young Investigator Award, the American Automatic Control Council Donald P. Eckman Award, and IFAC High Impact Paper Award, and he is a Fellow of IEEE and IFAC. Shamma is the Editor-in-Chief for the IEEE Transactions on Control of Network Systems and an associate editor of the IEEE Transactions on Robotics. 